\title{
早期康复护理干预对脑出血患者手术后偏瘫肢体功 能恢复的临床效果观察
}

\section{Clinical effect of early rehabilitation nursing intervention on the recovery of hemiplegic limb function in patients with cerebral hemorrhage after operation}

\author{
黄安 \\ An Huang \\ 江苏省邳州市中医院 中国・江苏 䂙州 221300
}

Pizhou Traditional Chinese Medicine Hospital Pizhou City, Pizhou, Jiangsu, 221300, China

\begin{abstract}
摘 要: 目的: 针对早期康复护理工作对于脑出血患者在手术后偏痽肢体的功能恢复情况进行研究,分析恢复效果。方法: 选取在 2018 年 3 月到 2020 年 3 月在我院脑外科就诊的 60 例脑出血患者,并通过随机分组的方法将该 60 例患者分为观察组 和对照组。对照组采用的是常规护理模式, 而观察组则采用常规护理模式加上早期康复护理干预,并以一个月为基准来对两 组患者进行手术后偏痽肢体功能恢复效果的评价, 采用百分制来进行打分。评价时以 FMA 、ADL 评分来作为比较指标。结 果: 通过为期一个月的护理工作可以得到, 观察组的脑出血患者 FMA 评分和 ADL 评分要明显的高于对照组, 并且两组的差异 存在统计学意义, 也就是 $\mathrm{P}<0.05$ 。结论: 仅仅采用常规护理方法对脑出血患者手术后偏痽肢体功能恢复效果并不显著, 而在 常规护理方法基础上添加早期康复护理可以获得较好的脑出血患者手术后偏痽肢体功能恢复效果,进而避免落残的情况,提 高了患者的生活质量,值得在临床上推广。
\end{abstract}

\begin{abstract}
Objective: To study the functional recovery of hemiplegic limbs of patients with cerebral hemorrhage after surgery for early rehabilitation nursing work, and analyze the recovery effect. Methods: Sixty patients with cerebral hemorrhage who were treated in the Department of Brain Surgery in our hospital from March 2018 to March 2020 were selected, and the 60 patients were randomly divided into observation group and control group. The control group adopts the conventional nursing model, while the observation group adopts the conventional nursing model plus early rehabilitation nursing intervention, and uses one month as the benchmark to evaluate the effect of the recovery of hemiplegic limb function after surgery in the two groups, using a percentile system. Score. In the evaluation, FMA and ADL scores were used as comparative indicators. Results: Through one-month nursing work, the FMA score and ADL score of cerebral hemorrhage patients in the observation group were significantly higher than those in the control group, and the difference between the two groups was statistically significant, that is, $\mathrm{P}<0.05$. Conclusion: The effect of conventional nursing methods on the recovery of hemiplegic limb function after cerebral hemorrhage surgery is not significant, and the addition of early rehabilitation care on the basis of conventional nursing methods can achieve better results in the recovery of hemiplegic limb function after cerebral hemorrhage surgery. It avoids disability, improves the quality of life of patients, and is worthy of clinical promotion.
\end{abstract}

关键词: 早期康复护理;脑出血患者;偏痽肢体功能;恢复效果

Keywords : early rehabilitation care; patients with cerebral hemorrhage; limb function of hemiplegia; recovery effect

DOI : $10.36012 /$ pmr. v2i6. 2973

【作者简介】黄安 (1986 ), 女, 汉, 江苏邳州人,主管护师, 本科学士学位, 从事临床护理研究。 


\section{1 前言}

脑出血对于中老年人的身体健康危害极大, 一旦犯病将 直接影响到患者的生命健康安全, 并且在治疗及康复过程患 者将大大的降低自身的自理能力, 甚至有些人完全丧失自理 能力。进几年, 随着脑出血患者年轻化趋势的加快, 该病已 经成为了危害人们生命健康的一大严重的疾病, 具统计有 40\%的患者在患病后落下终生残疾, 为家庭和生活带来了巨 大的灾难。所以为了能够提高脑出血患者在术后偏㿈肢体 功能的恢复率, 护理工作就显得尤为重要。现阶段, 患者术 后恢复期有效康复措施的研究已经成为了我国社会的一大 需求, 为了能够更好的探究脑出血患者术后恢复期的康复措 施, 本研究选取我院脑外科的 60 例脑出血患者案例作为研 究对象, 并针对患者在脑出血术后恢复期应用肢体康复训 练,分析其具体的康复效果, 研究的具体内容如下:

\section{2 资料与研究方法}

\section{1 研究资料}

本研究中选取 2018 年 3 月到 2020 年 3 月在我院脑外科 采取治疗措施的 60 例脑出血患者。本研究选取的患者全部 经过临床诊断和医学影像等技术, 确诊为脑出血患者, 患者 的意识清晰且并无其他精神方面问题, 其各项生命体征符合 脑出血患者的临床病征。本研究在选取脑出血患者时排除 具有精神疾病、心肚病、癌症、肿瘤、意识不清等能够严重影 响到本研究结果疾病的患者。本研究选取的患者都经过自 己或者家属代签协议同意, 属于自愿性参与医学研究, 并在 研究期间配合良好, 无不良反应和不良心态。本研究所选取 的患者中有 36 例男性患者, 24 例女性患者, 年龄分布为 45 岁到 81 岁。按照随机数表来将 60 例脑出血患者分为观察 组和对照组, 每组有 30 位患者。其中, 对照组 30 名成员中 有 21 名男性和 9 名女性,年龄分布在 45-80 岁, 平均年龄在 (65.3 44.6$)$ 。其中左侧偏㿈为 9 名, 右侧偏痽为 21 名; 观察 组 30 名患者中有 22 名男性和 8 名女性, 年龄分布在 50-81, 平均年龄在 $(65.6 \pm 4.7)$ 岁, 其中左侧偏㿈为 12 名, 右侧偏痽 为 18 名。根据统计学原理, 对照组和观察组的患者个人资 料与实际病情之间的差异并无统计学差异, 具有研究 性质 ${ }^{[1]}$ 。

\section{2 研究方法}

本研究中对 60 例脑出血术后偏㿈患者采取基础肢体康 复模式,该模式的具体内容如下:
(1)基础护理。每日做早间和晚间护理工作,对病患的血 压、呼吸、排便等多方面体征参数进行监测。首先要对患者 的皮肤情况进行检查，确保患者没有并发症。同时做好皮肤 护理,对患者频繁进行翻身等护理行为, 并确保患者的贴身 衣物、床上用品、医疗器具的清洁卫生。护理人员对偏㿈部 位采取按压措施来进行按摩,并在必要的时候涂抹鞣酸软 亳。其次要对患者的肌体状态进行分析, 确定患者的疼痛性 质, 并分析患者的运动状况和情绪精神状态, 对患者对疼痛、 心理状态进行分级和评价, 采取疏导服务。为了能够确保患 者的个人卫生健康, 需要定期对患者清洗会阴部位、肚门等, 昏迷患者必要时采取无菌导尿措施,放置导尿管或者是冲洗 膀胱。护理人员定期为患者检查尿常规和尿培养, 一旦发现 感染情况尽早使用抗生素。最后, 护理人员需要对患者的肠 胀气、便秘等情况进行处理,合理安排饮食, 如患者出现便秘 的症状可以进行艾负治疗, 每日一次, 取穴为: 神阙穴, 以补 中益气,温肠止泻,告知患者注意腹部保暖,勿食生冷刺激食 物, 并注意腹部及肚周皮肤情况。护理过程中主其注意患者 能够避免受凉引发肺炎的情况, 尽量帮助患者排出痰液。护 理人员在护理偏痽患者的过程中严格注意跌倒、汤伤等可能 会对患者肢体造成巨大生理伤害的情况,着重做好护栏保护 工作, 积极鼓励和帮助患者定期进行躯干的功能训练, 预防 肢体畸形 ${ }^{[2]}$ 。

(2)健康宣教。宣教对象主要面对患者以及患者家属, 对 二者讲述脑出血术后偏㿈的相关知识以及预防相应并发症、 提高康复效率的措施, 确保家属能够积极配合护理和康复工 作。护理人员要教授患者和患者家属对肢体功能恢复训练 的具体方法, 并嘱咐患者家属每日对患者肢体关节进行按 摩, 并对肢体进行保护, 防止其关节的傈硬与肌肉萎缩的情 况发生。护理人员对家属和病患讲述日常生活起居中需要 严格注意的问题,包括禁用热水袋等注意事项,并帮助患者 培养良好的生活习惯, 保持良好的生活作息 ${ }^{[3]}$ 。

(3)心理护理。心理护理工作包括与患者谈话、安排娱乐 活动等舒缓患者心态, 保持患者的心情愉快。

本研究中观察组的护理措施是以上述基础护理模式为 基础,加上早期康复护理措施, 具体包括四个方面的内容:

(1)心理康复的引导: 心理康复在患者的护理工作中有着 至关重要的作用,护理人员要定期巡查和询问患者的情绪状 态,分析患者是否存在有焦虑、抑郁等负面情绪, 并时刻记录 患者的心理状态变化,对患者加强心理上的辅导。护理人员 时常与患者和患者家属进行沟通谈话, 多给予患者在心理和 
身体上的关心与鼓励, 要保证家属能够以更加乐观的状态来 面对患者,这样才能够提高患者的治疗积极性, 使得患者在 精神和心理上能够受到更多的鼓励, 确保患者能够更有信心 的去配合医院治疗与护理,从而保障后续的康复。

(2)脑水肿期的护理: 在脑水肿期,护理人员需要每天对 观察组患者采取翻身拍背的护理措施,该过程需要保持在每 天两小时左右, 并在病情稍微稳定之后则开始康复护理措 施。首先, 护理人员需要保证患者能够在舒服的身体姿势下 采用超声联合及其 $\mathrm{B} 1+\mathrm{C} 1$ 配合治疗, 并且使用中频穴位治 疗仪配合中药穴位贴敷来帮助患者更好的预防在偏痽肢体 一侧的肌肉弯缩情况, 该措施取穴为: 患侧肩髃、曲池、外关、 足三里、三阴交、阳陵泉, 要保证每天治疗 1-2 次, 以达到疏 通经络, 活血化瘀, 改善肢体无力情况。其次就是护理人员 需要帮助患者进行在偏痽肢体以此的关节屈伸运动, 该措施 要保证每天可以进行 3-4 次,并配合以对肌肉的按摩。在护 理人员开展护理措施的过程中, 其动作应该要保证温柔轻 巧, 不能够过于用力, 要以病人的实际情况为主, 否则容易造 成二次伤害而加重患者的病情, 针对高血压患者, 可以进行 耳穴埋籽治疗, 每周两次, 取穴为: 降压沟、肾、神门、内耳, 以 刺激耳穴, 理气降压, 并指导患者正确按压耳穴的方法,保持 心情舒畅,监测血压变化。

(3)稳定期的护理: 稳定期相较于前两个阶段的护理工作 要轻松很多,在患者进入的到稳定期后已经获得了一定程度 的康复, 康复护理也可以进入到下一阶段。护理人员将患者 从躺卧姿势改变成坐姿,并且保证患者可以主动开始康复训 练, 鼓励患者自己锻炼一些康复动作, 包括翻身、起坐等姿 势。同时, 护理人员应该要叮嘱患者家属帮助患者进行康复 训练, 使用健手带等设备来对肢体进行伸展,包括外展、内 收、前屈等多个动作。

(4)恢复期的护理: 恢复期的护理则是在稳定期护理工作 获得一定的成果之后再开展的护理措施, 也就是该阶段的护 理措施建立在稳定期的护理之上,让患者开始站立训练、坐 起训练、步行训练, 并且逐渐加强患者的肢体负重能力, 让患 者可以主动的开始肢体训练。护理人员帮助患者使用其正 常躯体的力量来上举偏瘫肢体 ${ }^{[4]}$ 。

\section{3 观察指标}

观察观察组和对照组在护理工作前后的偏痽肢体恢复 效果, 并采用简式运动功能评分法 FMA 对患者的肢体运动 恢复情况进行评价, 采用 Barthel 指数 ADL 对患者的日常生 活能力改善情况进行评价。

\section{4 统计学方法}

本研究采用 SPSS 16.0 统计学软件来将两组护理数据 进行处理和分析, 采取 $\mathrm{t}$ 检验分析对照组和观察组的定量资 料。计数资料采用卡方检验, 在 $\mathrm{P}$ 小于 0.05 时具有统计学 意义。

\section{3 结果}

对照组和观察组在护理工作前后的 FMA 与 ADL 评分结 果的比较结果:

根据相应的统计方法, 观察组在采取早期康复护理干预 措施后期 FMA 评分与 ADL 评分都要高于对照组, 其中存在 的差异具有统计学意义, 也就是 $\mathrm{P}$ 小于 0.05 , 具体数据如 下表：

表 1 对照组和观察组的 FMA 与 ADL 评分比较

\begin{tabular}{c|c|c|c|c}
\hline \multirow{2}{*}{ 组别 } & \multicolumn{2}{|c|}{ FMA 评分 } & \multicolumn{2}{c}{ ADL 评分 } \\
\cline { 2 - 5 } & 护理前 & 护理后 & 护理前 & 护理后 \\
\hline 对照组 & $30.2 \pm 3.1$ & $45.1 \pm 6.7$ & $34.1 \pm 3.5$ & $50.1 \pm 4.6$ \\
\hline 观察组 & $30.8 \pm 5.2$ & $70.5 \pm 7.8$ & $27.6 \pm 5.2$ & $70.6 \pm 6.9$ \\
\hline
\end{tabular}

由上述数据可以证明,在脑出血患者术后偏㿈康复工作 中采用早期康复护理干预手段可以有效的促进患者的肢体 功能恢复, 患者的肢体运动恢复情况与日常生活能力改善情 况都有较大的提升, 相比于对照组来说, 观察组在应用了早 期康复护理之后有着更好的康复效果, 这足以证明在早期康 复护理干预措施可以在临床中使用 ${ }^{[5]}$ 。

\section{4 讨论}

根据相应的报道, 脑出血患者在术后虽然会存在偏痽等 情况, 严重影响其生活状态, 但是因为其中枢神经系统结构 和功能依然可以有效的展开重组,有较好的可塑性, 所以在 此时开展早期康复治疗措施可以有效的降低并发症的发生 几率,提高护理效果, 对患者的偏瘫、知觉障碍、运动障碍的 处理与恢复都有非常好的效果,配合常规治疗与护理措施可 以达到较好的康复效果,使得患者的肌体获得更好的恢复, 在临床上具有非常好的推广意义 ${ }^{[6]}$ 。

\section{5 结束语}

综上所述, 本文探讨了关于脑出血患者在手术后偏痽肢 体功能恢复护理中采用早期康复护理措施的可行性,并通过 研究证明了使用了早期康复 (下转第 43 页) 
况下进行多次检查。在临床上可以使用 $24 \mathrm{~h}$ 尿白蛋白定量 或者是尿蛋白排泄率来进行对蛋白尿的评估。其次是肾小 球滤过率, 其在检测中主要注意以下两点问题。首先是内生 肌酮清除率受到的印象因素较多, 需要使用无菌容器等来提 高内生肌酐清除率的检测准确性。其次就是可以通过基于 血肌酐的公式来计算内生肌酐清除率, 该方法虽然效果较 好, 但是会包含患者的性别和年龄等影响参数, 可能会在后 续建模研究中消弱性别和年龄对模型的贡献。另外还可以 使用肾动态评估法或者其他应用外源性标志物评估法来进 行测量。

\section{5 诊断和透析}

对于慢性肾脏病的分期诊断工作来说, 因为慢性肾脏病 实际上是一类病的总称, 其是一种临床综合征的总和, 所以 在临床上应该要针对于其背后的主要原因进行分析, 将各种 原因占比进行计算, 明确慢性肾脏病的病因之后才能够做到 对症下药。在治疗上, 治疗慢性肾脏病的本质要点就在于延 缓慢性肾脏病过渡到终末期肾脏病的过程, 并且对并发症的 治疗、合并症的治疗等都是具体的治疗手段,这一点需要医 务人员能够牢记, 切勿本末倒置 ${ }^{[6]}$ 。

对于多项研究表明了患者在进入透析时其内生肌酐清除率 的水平越高, 患者就面临着更多的生命危险, 然而实际上并 没有确切的证据证明了在进入透析时内生肌酤清除率对患 者致死率的影响, 其还是需要针对具体的情况进行分析。根 据现阶段肾脏病预后质量指南得到, 在内生肌酐清除率达到 10 以下就可以采用透析的方式进行治疗, 但是患者如果生 命体征较为良好, 其尿量稳定且无水肿的情况发生, 营养状
况也比较均衡, 那公可以暂时不用做透析。而对于存在腹 泻、感染情况的患者, 哪怕其内生肌酤清除率达到 10 以下还 是依然要做透析治疗。

\section{4 结束语}

综上所述,本文探讨了关于慢性肾脏病在管理中的几点 问题,提出了几点管理措施。可以说, 据现阶段来看, 我国医 院必须要针对慢性肾脏病进行进行管理,建立起完善的管理 机制和规范, 确保旧患者能够有效的减缓在慢性肾脏病到终 末期肾脏病的过程, 提高在管理中的科学性和规范性, 减少 随意性,从而更好的保证患者的就诊质量和康复效果。

\section{参考文献}

１］滒春艳, 王牡丹, 谌清玲, 王玉婷, 杨琴. 健康管理平台在慢性 肾脏病患者自我管理中的应用 $[\mathrm{J}]$. 中华现代护理杂志, 2019 (06) :677-681.

[2] 林雅慧, 刘玉红, 王宓, 左力. 社区高危人群慢性肾脏病患病率 及節查策略研究 $[\mathrm{J}]$. 中国实用内科杂志, 2018,38(12):1169 -1172 .

[3] 纪星星, 梅玉秀, 程霞, 简桂花. 慢性肾脏病管理现状的探讨 $[\mathrm{J}]$. 中外女性健康研究, 2017 (10) : 12-14.

４］］施月仙. 慢性肾脏病患者矿物质和骨异常的非疾病性相关因 素和干预效果的研究 [D]. 天津医科大学,2016.

[5] 中华内分泌代谢杂志 2011 年第 27 卷主题词索引 $[\mathrm{J}]$. 中华内 分泌代谢杂志, 2011 (12): 1040-1057. [6]临床糖尿病学 [M]. 浙江工商大学出版社: , 201405. 252.
(上接第 38 页) 护理措施的观察组在肢体功能和生活能力方 面相比对照组都有较大的改善,所以早期康复护理干预方法 可以在脑出血术后偏瘫肢体功能恢复工作中有较好的应用 价值, 在临床上值得推广。

\section{参考文献}

[1] 王静, 唐瑶, 胡霞. 早期康复护理对脑出血患者手术后偏瘫肢 体功能恢复的临床效果观察 $[\mathrm{J}]$. 特别健康, 2020, ( 1 ):212 -213 .

[2] 苒维, 手燕. 早期康复护理对脑出血患者手术后偏痽肢体功能 恢复的影响分析 $[\mathrm{J}]$. 饮食保健, 2019,6(25): 117.
[3] 曹静. 早期康复护理对脑出血患者手术后偏瘫肢体功能恢复 的影响 $[\mathrm{J}]$. 医疗装备, 2017,30(7): 186-187. DOI: 10. 3969/j. issn. 1002-2376. 2017. 07. 125.

[4] 吴东燕. 脑出血手术后偏瘫应用早期康复护理干预的肢体功 能恢复效果研究 $[\mathrm{J}]$. 心理医生,2016,22(21) : 178-178,179.

[5]王维. 早期康复治疗在脑出血偏痽患者临床治疗中的应用效 果分析 [J]. 养生保健指南, 2018, ( 1 ) : 71. DOI: 10. 3969/j. issn. 1006-6845. 2018. 01. 062.

[6] 苗丹丹. 对接受手术治疗的脑出血患者进行早期康复护理的 效果探讨 $[\mathrm{J}]$. 当代医药论丛, 2020,18(4):282. 